\title{
Defibrotide - A New Treatment Approach for Severe Veno-occlusive Disease
}

\author{
Katrina Mountfort ${ }^{1}$ \\ Expertly Reviewed by the Symposium Speakers: Mohamad Mohty ${ }^{2,3}$ and Elisabeth Wallhult ${ }^{4}$ \\ 1. Senior Medical Writer, Touch Medical Media, Reading, UK; 2. Professor of Hematology, Saint-Antoine Hospital, Paris, France; \\ 3. Head of the Hematology and Cellular Therapy Department, University Pierre and Marie Curie, Paris, France; 4. RN and Quality Manager, \\ Section of Haematology and Coagulation, Sahlgrenska University Hospital, Gothenburg, Sweden
}

\begin{abstract}
Severe veno-occlusive disease (VOD) is a serious and life-threatening complication of haematopoietic stem cell transplantation (HSCT), for which the standard of care has until recently been supportive care. VOD is the result of a primary injury to sinusoidal endothelial cells and severe VOD is characterised by sinusoidal narrowing and occlusion, which leads to portal hypertension, multi-organ failure (MOF) and, ultimately, death. Defibrotide regulates multiple pathways involved in the pathological processes underlying VOD and is the first drug to be approved in Europe for the treatment of severe VOD. Defibrotide is indicated for the treatment of severe hepatic VOD in HSCT therapy in adults and infants aged over 1 month. A phase III study found significant increases in complete response (CR) and survival with defibrotide compared with historical controls. These data together with earlier studies and an ongoing expanded access protocol in a large patient cohort demonstrate improved outcomes with defibrotide in severe VOD and highlight the importance of treatment with defibrotide.
\end{abstract}

\section{Keywords}

veno-occlusive disease, defibrotide, haematopoietic stem cell transplantation

Disclosure: Katrina Mountfort is an employee of Touch Medical Media. Mohamad Mohty received research support and lectures honoraria from Gentium/JAZZ Pharmaceuticals whose product is discussed in this manuscript. Elisabeth Wallhult has received honorarium from Gentium/Jazz Pharmaceuticals for participation in this symposia and has no other disclosures or conflicts of interest to declare.

open Access: This article is published under the Creative Commons Attribution Noncommercial License, which permits any non-commercial use, distribution, adaptation and reproduction provided the original author(s) and source are given appropriate credit.

Received: 5 December 2014 Accepted: 4 March 2015 Citation: European Oncology \& Haematology, 2015;11(1):11-4 DOl: 10.17925/EOH.2015.11.01.11 Correspondence: Katrina Mountfort, Touch Medical Media, The White House, Mill Road, Goring-on-Thames, RG8 9DD, UK. E: katrina.mountfort@touchmedicalmedia.com

Support: This report is based on two satellite symposia and a press event supported by Gentium at the 40th Annual Meeting of the European Society for Blood and Marrow Transplantation meeting in Milan, Italy on 30 March to 2 April 2014. The publication of this report and medical writing assistance was supported by Jazz Pharmaceuticals.

Haematopoietic stem cell transplantation (HSCT) has become the standard of care for many haematological malignancies, selected solid tumours and some non-malignant disorders. ${ }^{1}$ However, it can be associated with serious complications, in particular, veno-occlusive disease (VOD). Approximately $14 \%$ of HSCT patients develop VOD, although incidences of up to $60 \%$ have been reported..$^{2,3}$ Incidence is higher in children and is partly due to certain malignant and inherited diseases that are associated with a substantially increased risk of VOD during HSCT. ${ }^{4}$ VOD can also occur in cancer patients undergoing aggressive chemotherapy regimens and has been reported after solid organ transplantation. VOD places a significant burden on healthcare providers and can add considerable additional expense to HSCT costs. ${ }^{5}$ VOD presents with different spectrums of severity, from mild VOD, which is self-limiting and requires no treatment, to severe VOD, which is associated with a mortality of over $80 \%$.,6

In October 2013, the European Commission granted marketing authorisation for defibrotide (Defitelio ${ }^{\circledR}$ Gentium SpA) for the treatment of severe hepatic VOD in HSCT therapy in adults and infants aged over 1 month. Defibrotide is the first drug to be approved for severe VOD in Europe. In context with the launch of Defibrotide in Europe, two satellite symposia and a press event were held at the 40th Annual Meeting of the European Society for Blood and Marrow Transplantation (EBMT) on the 31st March and 1st April 2014 in Milan, Italy. This article, based on presentations at the EBMT meeting, will describe the pathophysiology, signs and symptoms of VOD and the use of defibrotide in the treatment of severe VOD.

\section{Clinical Manifestations of Veno-occlusive Disease}

Severe VOD is defined as VOD with the presence of multi-organ failure (MOF). ${ }^{2}$ This can include pulmonary failure (oxygen saturation $\left[\mathrm{SO}_{2}\right]$ $<90 \%$ and/or ventilator dependence); renal failure (doubling of serum creatinine and/or dialysis dependence) and central nervous system (CNS) abnormalities (confusion, encephalopathy and coma). Symptoms of VOD can include painful hepatomegaly, jaundice (serum bilirubin $\geq 2 \mathrm{mg} / \mathrm{dl}$, $34.2 \mathrm{mmol} / \mathrm{l})$, fluid retention, weight gain ( $\geq 5 \%$ ), ascites, usually with onset in the first 3-4 weeks following HSCT, and the absence of other causes. ${ }^{4,7}$ Certain clinical signs of VOD, including a rapid increase of bilirubin level 


\section{Figure 1: Pathophysiology of Veno-occlusive Disease}

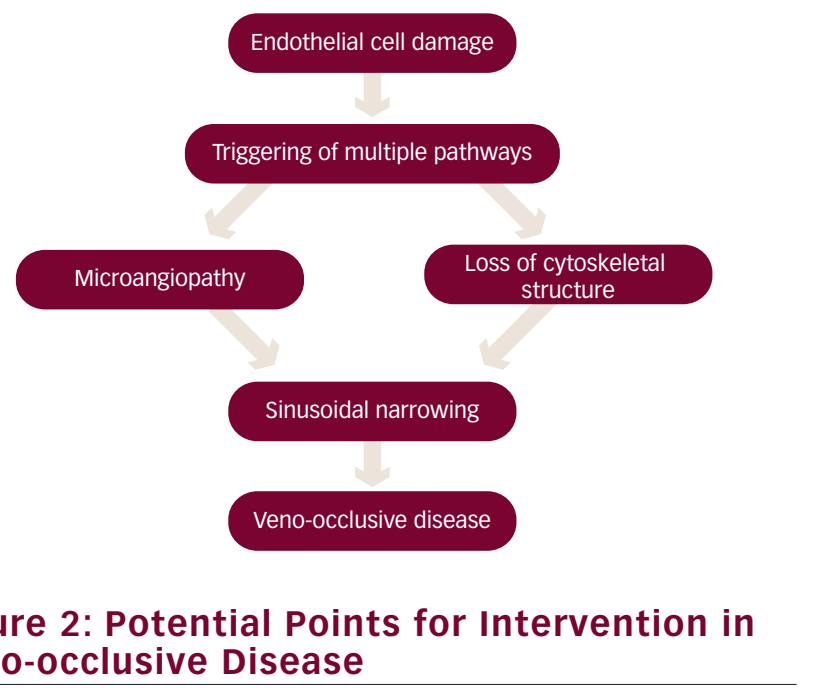

\section{Figure 2: Potential Points for Intervention in Veno-occlusive Disease}

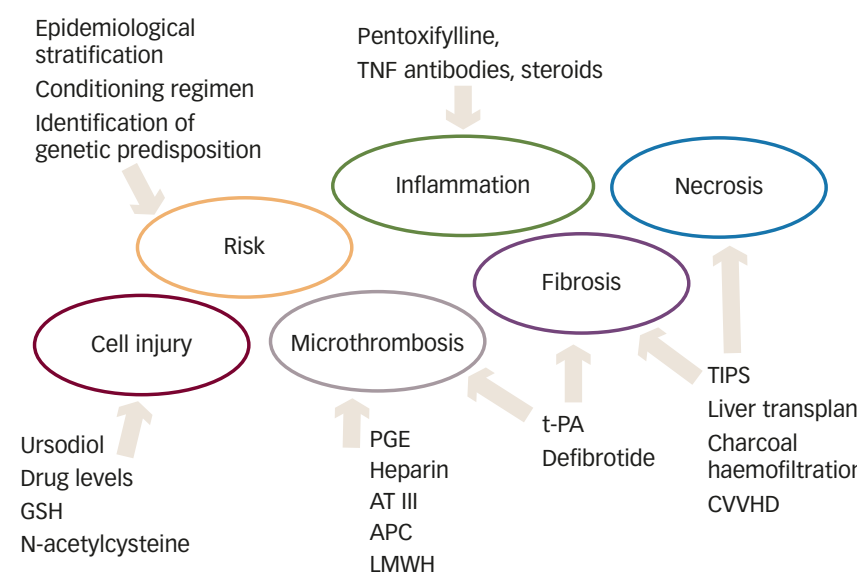

$A P C=$ activated protein $C: A T I I I=$ antithrombin III; CWHD = continuous veno-venous haemodialysis; $G S H=$ glutathione; $L M W H=$ low molecular weight heparin; $P G E$ prostaglandin E; TIPS = transjugular intrahepatic portosystematic shunt; TNF = tumour necrosis factor; $t$-PA = tissue plasminogen activator. Adapted from: Richardson et al., $2001 .{ }^{20}$

and rapid weight gain, are associated with a poor prognosis. ${ }^{8}$ Early signs of MOF, including oxygen requirement, renal dysfunction and encephalopathy are, to date, strong predictors of a fatal outcome.2

Risk factors for VOD may be patient- or transplant-related. General risk factors include extremes of age (children and the elderly) and low Karnofsky score. Underlying disease is also important: an absence of malignancy is associated with lower risk than malignancy, and remission is lower risk than active disease. Previous liver radiation is a risk factor, as are alterations in liver status (e.g. fibrosis, cirrhosis). Other risk factors include acute hepatitis at HSCT and previous treatment with gemtuzumab ozogamicin, a monoclonal antibody used in the treatment of acute myelogenous leukaemia. ${ }^{9-12}$ Risk factors in paediatric patients include younger age. ${ }^{13}$ Transplant-related risk factors include highdose conditioning regimens, allogeneic rather than autologous HSCT, the stem cell source (bone marrow-derived stem cells are associated with higher risk than peripheral-derived), a second HSCT, abdominal irradiation, use of busulphan-based conditioning regimens and the use of hepatotoxic drugs (e.g. progestogens, calcineurin inhibitors). ${ }^{10}$ Risk factors should be considered prior to HSCT.

Diagnosis of VOD should be based primarily on established clinical criteria. Clinical criteria for diagnosis have been determined by two separate groups: the Seattle and Baltimore criteria. ${ }^{14}$ The modified Seattle criteria require presentation before day 20 post-HSCT of two of the following: bilirubin $>2 \mathrm{mg} / \mathrm{dl}(\sim 34 \mu \mathrm{mol} / \mathrm{l})$, hepatomegaly or right upper quadrant pain of liver origin or unexplained weight gain of $>2 \%$ baseline due to fluid accumulation. ${ }^{6}$ The Balitimore criteria require bilirubin $\geq 2 \mathrm{mg} /$ dl ( $\sim 34 \mu \mathrm{mol} / \mathrm{l})$ before day 21 post-HSCT and at least two of the following: hepatomegaly, ascites and weight gain $\geq 5 \%$ from baseline. ${ }^{15}$

\section{Pathophysiology of Veno-occlusive Disease}

The pathophysiological cause of VOD is a primary injury to sinusoidal endothelial cells, hepatocytes or stellate cells, producing a prothrombotic phenotype at the endothelial cell surface. ${ }^{4}$ Endothelial activation and damage after HSCT occurs in both the allogeneic and autologous setting and affects both microvascular and macrovascular endothelial cells. ${ }^{16}$ Endothelial damage can be seen at the early stages of HSCT, induced by a number of factors including the conditioning regimen, pro-inflammatory agents used during transplantation, the translocation of endotoxins across the damaged gastrointestinal tract and the engraftment. ${ }^{17}$ The resulting activation and damage to the sinusoidal endothelium triggers multiple pathways: resident Kupffer cells express cytokines and markers of endothelial damage, including tumour necrosis factor (TNF)-alpha; adhesion molecules including intercellular adhesion molecule 1 (ICAM1), the expression of which is associated with inflammation in the blood vessel wall, and vascular adhesion molecule 1 (VCAM-1); plasminogen activator inhibitor 1 (PAl-1), an important inhibitor of fibrinolysis; von Willebrand factor, a vital element of cell surface coagulopathy and platelet aggregation; tissue factor (TF); tissue-plasminogen activator (t-PA); and heparanase, an enzyme responsible for breaking down extracellular barriers contributing to extracellular matrix integrity and thereby tissue homeostasis. Subsequent endothelial cell rounding and gap formation allows the diffusion of red blood cells, leukocytes and other debris into the space of Disse, which separates the endothelial cells and hepatocytes. The accumulation of these cells and debris leads to fibrin deposition and occlusion of the small pores that perforate the endothelial lining. Endothelial cells subsequently dissect off and embolise downstream, contributing to sinusoidal narrowing. ${ }^{4}$

VOD is characterised by a procoagulant and hypofibrinolytic state, which contributes to fibrin deposition, increased clot formation and reduced clot breakdown, obstructing the sinusoids. The consequences of these events are reduced hepatic venous outflow and, in severe disease, MOF and death (see Figure 1)..$^{11,18}$

\section{Treatment and Management of Veno-occlusive Disease}

Until recently, there has been no established and approved treatment for VOD and the standard of treatment has been best supportive care, particularly in the management of fluid balance.11,13 Management of VOD requires supportive measures. First-line therapy should consist of diuresis; management of sodium and fluid intake; and maintenance of intravascular volume and renal perfusion by means of albumin, plasma expanders and transfusions. Other symptomatic treatments include haemofiltration and haemodialysis, analgesia, paracentresis/ thoracocentresis and mechanical ventilation. ${ }^{4,11}$ Transjugular intrahepatic portosystemic shunt (TIPS) has been used occasionally in severe VOD complicated by portal hypertension, but overall survival is limited. ${ }^{19}$

Therapeutic approaches to VOD should aim to modulate endothelial cell injury without causing systemic bleeding or other toxicities, as well as protecting the host without compromising the anti-tumour effect 


\section{Table 1: Clinical Trials Investigating the Safety and Efficacy of Defibrotide in Severe Veno-occlusive Disease}

\begin{tabular}{|c|c|c|c|c|c|}
\hline Trial & Design & Key Endpoints & Other Endpoints & Safety & Reference \\
\hline $\begin{array}{l}\text { Phase I } \\
\text { retrospective review }\end{array}$ & $\begin{array}{l}\mathrm{n}=19 \text {, compassionate use; } 5-60 \mathrm{mg} / \mathrm{kg} / \text { day } \\
\text { (intra-patient dose escalation, until response/toxicity) }\end{array}$ & CR: $42 \%$ & $\begin{array}{l}\text { Day }+100 \text { survival: } \\
32 \%\end{array}$ & $\begin{array}{l}\text { Minimal toxicity at } \\
\text { doses tested }\end{array}$ & 29 \\
\hline Phase I/II & $\begin{array}{l}\mathrm{n}=88 \text { (including } 19 \text { patients from the retrospective } \\
\text { analysis), emergency use; DF: 10-60 mg/kg/day } \\
\text { (intra-patient dose escalation, until response/toxicity) }\end{array}$ & CR: $36 \%$ & $\begin{array}{l}\text { Day }+100 \text { survival: } \\
35 \%\end{array}$ & $\begin{array}{l}\text { No serious AES } \\
\text { attributed to DF }\end{array}$ & 30 \\
\hline Phase II & $\begin{array}{l}n=149 \text {, randomised, dose-finding; Arm A: DF } \\
25 \mathrm{mg} / \mathrm{kg} / \text { day }(\mathrm{n}=75) ; \text { Arm B: DF } 40 \text { mg/kg/day } \\
(\mathrm{n}=74) \text { for } 14 \text { days or more }\end{array}$ & $\begin{array}{l}\text { Day +100 CR: } \\
46 \% \text { effective } \\
\text { dose } 25 \mathrm{mg} / \mathrm{kg} / \text { day }\end{array}$ & $\begin{array}{l}\text { Day +100 survival: } \\
42 \%\end{array}$ & $\begin{array}{l}\text { Overall AE incidence: } \\
8 \% \text { (greater at } 40 \text { versus } \\
25 \mathrm{mg} / \mathrm{kg} / \text { day) }\end{array}$ & 32 \\
\hline Phase III & $\begin{array}{l}\mathrm{n}=102 \text { (DF); } \mathrm{n}=32 \text { (HC) non-randomised, } \\
\text { comparison to HC; DF: } 6.25 \mathrm{mg} / \mathrm{kg} \text { IV q6h } \\
\text { (25 mg/kg/day) for } 21 \text { days or more }\end{array}$ & $\begin{array}{l}\text { Day +100 CR: } \\
\text { DF } 24 \% ; \text { HC } 9 \% \\
(p=0.0131)\end{array}$ & $\begin{array}{l}\text { Day }+100 \text { mortality: } \\
\text { DF } 62 \% ; \text { HC } 75 \% \\
(p=0.0341)\end{array}$ & $\begin{array}{l}\text { Haemorrhagic AEs: DF } \\
65 \% \text {; HC } 69 \% \\
\text { Generally well-tolerated }\end{array}$ & 33 \\
\hline
\end{tabular}

$A E S=$ adverse events $; C R=$ complete response $; \mathrm{DF}=$ defibrotide; $H C=$ historical control.

of cytotoxic therapy. There are several potential areas for intervention in VOD (see Figure 2);20 however, few have led to treatment options with acceptable efficacy and safety. Studies investigating systemic anticoagulants and/or thrombolytics as treatment options for VOD have not shown survival benefit and demonstrated a risk of severe and often life-threatening bleeding complications. ${ }^{4}$ In patients with severe VOD who have already developed MOF, the combination of heparin and recombinant t-PA has been associated with a response of up to $30 \%$ of patients. However, overall survival was poor, and the treatment has demonstrated excessive toxicity, including an increased risk of lifethreatening bleeding, precluding the use of this combination in this population. ${ }^{49,21}$ Therefore, there remains a need for effective and safe therapies that target the underlying cause of severe VOD.

The Haemato-oncology Task Force of British Committee for Standards in Haematology (BCSH) and British society for Blood and Marrow Transplantation (BSBMT) ${ }^{14}$ recently published guidelines for the diagnosis and management of VOD. While defibrotide is only indicated for the treatment of severe hepatic VOD in HSCT therapy, these guidelines discuss the use of defibrotide for the treatment of VOD in adults and children. t-PA is not recommended for use in the treatment of VOD due to the associated risk of haemorrhage. $\mathrm{N}$-acetylcysteine is not routinely recommended due to lack of efficacy in VOD. Methylprednisolone may be considered for use in the treatment of VOD, though with caution regarding infection. Early discussion with critical care specialists and a specialist hepatology unit is also recommended.

\section{Introduction to Defibrotide}

Defibrotide is a complex mixture of oligonucleotides derived from porcine intestinal mucosa and is prepared by the controlled depolymerisation of DNA. ${ }^{4,18}$ Defibrotide is currently approved in the EU for the treatment of severe hepatic VOD in HSCT therapy. It is indicated in adults, adolescents, children and infants over 1 month of age. ${ }^{14}$

The mechanism of action of defibrotide has not yet been fully elucidated, but preclinical studies suggest that it binds to multiple sites on the vascular endothelium that are involved in cell regulation, including adenosine receptors, providing a stimulus that promotes the protection of activated endothelial cells. ${ }^{22}$ It also prevents endothelial damage in patients undergoing HSCT by modulating critical endothelial stress responses. ${ }^{23,24}$ Immunosuppressive drugs used in conditioning regimes in HSCT include cyclosporine (CSA), tacrolimus (TAC) and sirolimus (SIR); these induce an increased expression of adhesion receptors such as ICAM-1, as well as increasing the reactivity of the extracellular matrix to circulating platelets. Defibrotide attenuates these effects, ${ }^{24}$ protects endothelial cells from fludarabine (F-Ara)-mediated apoptosis ${ }^{25}$ and inhibits the expression of heparanase. ${ }^{26}$

In addition to its effects on endothelial cell protection, it is anticipated that defibrotide exerts antithrombotic effects and restores the thrombofibrinolytic balance by selectively increasing prostaglandin 12 and E2 levels, leading to increased tissue plasminogen activator expression in microvascular cells and decreased t-PA function. ${ }^{27}$ Defibrotide also inhibits TF and attenuates the increased expression of von Willebrand Factor seen after HSCT23 and has a weak profibrinolytic activity in vitro. ${ }^{28}$

\section{Clinical Trial Data}

Several studies show evidence of the efficacy and safety of defibrotide in severe VOD (see Table 1).29-32 A phase II dose-finding study established that no significant difference was seen in complete response (CR) or survival between patients treated with doses of 25 or $40 \mathrm{mg} / \mathrm{kg} / \mathrm{day}{ }^{32} \mathrm{As}$ a result, the current treatment regime was established, which consists of intravenous administration of defibrotide at $6.25 \mathrm{mg} / \mathrm{kg}$ every 6 hours (25 mg/kg/day), with recommended treatment duration of at least 21 days and continued until the symptoms and signs of severe VOD resolve.

The pivotal trial leading to EU approval of defibrotide was a historically controlled, multicentre, open-label phase III study that demonstrated the efficacy of defibrotide $25 \mathrm{mg} / \mathrm{kg} /$ day in patients with severe VOD. Due to the life-threatening nature of severe VOD with MOF, a trial randomising patients to placebo or best supportive care was rejected on the basis of ethical concerns. Patients treated with defibrotide $(n=102)$ had significantly increased CR and survival at day 100 compared with historical controls ( $n=32$ ): CR was $24 \%$ compared with $9 \%$ in historical controls; survival at day 100 was $38 \%$ compared with $25 \%$ in historical controls. In children, CR at day 100 was $36 \%$ versus $7 \%$ in historical controls; $p=0.04$. Haemorrhagic adverse events (AEs) were similar between treatment and control arms (65\% versus $69 \%$ ); $18 \%$ of treated patients experienced a drug-related toxicity that led to discontinuation. ${ }^{33}$

Defibrotide has been made available in the US since 2007 via a prospective treatment-investigational new drug (T-IND) expanded access protocol. This is to date the largest performed prospective evaluation of defibrotide for the treatment of VOD in patients undergoing HSCT and patients who developed VOD following chemotherapy (i.e. non-HSCT patients) and includes almost 300 patients with severe VOD. The study is ongoing and, to date, 659 patients are enrolled. The primary endpoint is $\mathrm{CR}$ defined as total bilirubin $<2 \mathrm{mg} / \mathrm{dl}$ and resolution of $\mathrm{MOF}^{31}$ 
A recent interim analysis of the T-IND expanded access protocol provides efficacy and safety data on 470 patients. Of the 470 patients, 425 had VOD post-HSCT and, of those patients, VOD was severe in 284. In this patient group, which reflects the product's licensed indication, CR was $29 \%$ and survival at day 100 was $48 \%$.

Data are also available on the entire post-HSCT cohort $(n=425)$, although it should be noted that this included 141 patients with non-severe VOD (33\%). These data demonstrate that children ( $\leq 16$ years) had higher CR rates (41\% versus $27 \% ; p=0.0038$ ) and survival (60\% versus $49 \% ; p=0.0203$ ) compared with adults, supporting the importance of this agent in the paediatric population in particular. ${ }^{31}$ The results seen in the severe VOD post-HSCT patients in T-IND are consistent with the defibrotide patients in the phase III study: CR of $29 \%$ and $33 \%$, respectively, and survival of $48 \%$ and $55 \%$, respectively.

Toxicity proved generally manageable: $21 \%$ of patients experienced AEs, primarily haemorrhage including pulmonary bleeding (6\%), gastrointestinal hemorrhage (7\%), epistaxis (3\%), haematuria (2\%) and hypotension (4\%), including defibrotide-related events leading to discontinuation in $15 \%$ of patients. The level of all-grade graft versus host disease (GVHD) in the allogeneic HSCT patients were low at $11 \%$.

The T-IND study continues to enrol patients at 75 centres across the US

\section{Future Directions in Defibrotide Research}

Data from studies of defibrotide in treating VOD may suggest that it may be a potential agent to prophylactically protect the endothelium from activation and damage in HSCT. A large, prospective open-label, phase III trial has investigated defibrotide for prophylaxis of hepatic VOD in paediatric HSCT. Patients $(n=356)$ receiving defibrotide had a lower incidence of VOD at 30 days post-HSCT (12 \% versus $20 \%$; $p=0.0488$ ) as well as a lower incidence and severity of GVHD. ${ }^{34}$ However, there is a need for additional prospective studies investigating the effect of earlier intervention, prior to the development of MOF. The BCSH/BSBMT guidelines recommend defibrotide prophylaxis in children undergoing allogeneic transplant with at least one risk factor (evidence level 1A). Defibrotide prophylaxis is also suggested in adults undergoing allogeneic transplant with at least one risk factor (evidence level 2B). ${ }^{14}$

Hints of a low incidence and severity of GVHD have been reported in HSCT patients receiving defibrotide. ${ }^{31,34}$ GVHD is a major complication following HSCT, and the role of defibrotide warrants further study. Defibrotide has received Orphan Drug Designation by both the US Food and Drug administration (FDA) and the European Medicine Agency (EMA) for the prevention of GVHD.

Based on its effects on endothelial injury/activation and on inflammation, defibrotide may also have potential indications in other syndromes characterised by endothelial activation and microangiopathy. Defibrotide is currently licensed in Europe for the treatment of severe hepatic VOD in HSCT therapy. It would be helpful to investigate the role of defibrotide in other high-risk groups.

\section{Summary and Concluding Remarks}

Severe VOD is a devastating and often fatal consequence of HSCT for which, until recently, supportive care was the only treatment option. Defibrotide is licensed for the treatment of severe hepatic VOD in HSCT therapy. The mechanism of action of defibrotide includes endothelial cell protection and restoration of the thrombo-fibrinolytic balance.

Defibrotide has consistently demonstrated efficacy and safety in numerous clinical trials, and the large ongoing T-IND study has confirmed these findings. Accurate and timely recognition of severe VOD is important since the data suggest that patients who receive defibrotide late in the course of the disease have poorer outcomes than those who receive it earlier. Defibrotide is generally well tolerated and a low incidence of defibrotide-associated toxicities has been reported.
1. Gratwohl A, Baldomero H, Aljurf M, et al., Hematopoietic stem cell transplantation: a global perspective, JAMA, 2010:303:1617-24.

2. Coppell JA, Richardson PG, Soiffer R, et al., Hepatic venoocclusive disease following stem cell transplantation: incidence, clinical course, and outcome, Biol Blood Marrow Transplant, 2010;16:157-68.

3. Corbacioglu S, Honig M, Lahr G, et al., Stem cell transplantation in children with infantile osteopetrosis is associated with a high incidence of VOD, which could be prevented W defibrotide, Bone Marrow Transplant, 2006;38:547-53

Ho VT, Linden E, Revta C, et al., Hepatic veno-occlusive disease after hematopoietic stem cell transplantation: review and update on the use of defibrotide, Semin Thromb Hemost, 2007;33:373-88

5. Saito AM, Zahrieh D, Cutler C, et al., Lower costs associated with hematopoietic cell transplantation using reduced intensity vs high-dose regimens for hematological malignancy, Bone Marrow Transplant, 2007;40:209-17.

6. McDonald GB, Hinds MS, Fisher LD, et al., Veno-occlusive disease of the liver and multiorgan failure after bone marrow transplantation: a cohort study of 355 patients, Ann Intern Med, 1993;118:255-67.

. Bearman SI, The syndrome of hepatic veno-occlusive disease after marrow transplantation, Blood, 1995;85:3005-20.

Bearman $\mathrm{SI}$, Anderson $\mathrm{GL}$, Mori M, et al., Venoocclusive disease of the liver: development of a model for predicting fatal outcome after marrow transplantation, $J$ Clin Oncol, 1993;11:1729-36.

9. Helmy A, Review article: updates in the pathogenesis and therapy of hepatic sinusoidal obstruction syndrome, Aliment Pharmacol Ther, 2006:23:11-25.

10. Kumar S, DeLeve LD, Kamath PS, et al., Hepatic veno-occlusive disease (sinusoidal obstruction syndrome) after hematopoietic stem cell transplantation, Mayo Clin Proc, 2003:78:589-98.

11. Carreras E, Early complications after HSCT In: Apperley t. Carreras E Gluckman E Masszi T, eds, EBMT-ESH Handbook of Carreras E, Gluckman E, Masszi T, eds, EBMT-ESH Handbook

12. Cheuk DK, Wang P, Lee TL, et al., Risk factors and mortality predictors of hepatic veno-occlusive disease after pediatric hematopoietic stem cell transplantation, Bone Marrow Transplant, 2007;40:935-44.

13. Cesaro S, Pillon M, Talenti E, et al., A prospective survey on incidence, risk factors and therapy of hepatic veno- occlusive disease in children after hematopoietic stem cell transplantation, Haematologica, 2005;90:1396-404.

14. Dignan FL, Wynn RF, Hadzic N, et al., BCSH/BSBMT guideline: diagnosis and management of veno-occlusive disease (sinusoidal obstruction syndrome) following haematopoietic stem cell transplantation, Br J Haemato I 2013;163:444-57.

15. Jones RJ, Lee KS, Beschorner WE, et al., Venoocclusive disease of the liver following bone marrow transplantation, disease of the liver following bo

16. Palomo M, Diaz-Ricart M, Carbo C, et al., The release of soluble factors contributing to endothelial activation and damage after hematopoietic stem cell transplantation is not limited to the allogeneic setting and involves several pathogenic mechanisms, Biol Blood Marrow Transplant, 2009;15:537-46.

17. Palomo M, Diaz-Ricart M, Carbo C, et al., Endothelial dysfunction after hematopoietic stem cell transplantation: role of the conditioning regimen and the type of transplantation, Biol Blood Marrow Transplant, 2010;16:985-93.

18. Richardson PG, Corbacioglu S, Ho VT, et al., Drug safety evaluation of defibrotide, Expert Opin Drug Saf, 2013;12:123-36.

9. Azoulay D, Castaing D, Lemoine A, et al., Transjugular intrahepatic portosystemic shunt (TIPS) for severe venoocclusive disease of the liver following bone marrow transplantation, Bone Marrow Transplant, 2000;25:987-92.

20. Richardson P, Guinan E, Hepatic veno-occlusive disease following hematopoietic stem cell transplantation, Acta Haematol, 2001;106:57-68.

21. Bearman SI, Lee JL, Baron AE, et al., Treatment of hepatic venocclusive disease with recombinant human tissue plasminogen activator and heparin in 42 marrow transplant patients, Blood, 1997;89:1501-6.

22. Bianchi G, Barone D, Lanzarotti E, et al., Defibrotide, a singlestranded polydeoxyribonucleotide acting as an adenosine receptor agonist, Eur J Pharmacol, 1993:238:327-34.

23. Palomo M, Diaz-Ricart M, Rovira M et al., Defibrotide prevents the activation of macrovascular and microvascular prevents the activation of macrovascular and microvascular autologous hematopoietic stem cell transplantation, Biol Blood Marrow Transplant, 2011;17:497-506.

24. Carmona A Diez-Ricart M, Palomo M et al Distinct deleterious effects of cyclosporine and tacrolimus and combined effects of cyclosporine and tacrolimus and combined defibrotide, Biol Blood Marrow Transplant, 2013;19:1439-45.
25. Eissner G, Multhoff G, Gerbitz A et al. Fludarabine induces apoptosis, activation, and allogenicity in human endothelia and epithelial cells: protective effect of defibrotide, Blood, 2002:100:334-40.

26. Echart C, lacobelli M, Mitsiades $C$, et al., Pre-clinical evidence for an anti-tumor activity of defibrotide, a DNA-based endothelium stabilizing drug I Clin Oncol, 2007:18S:141-44.

7. Palmer KJ, Goa KL, Defibrotide. A review of its pharmacodynamic and pharmacokinetic properties, and therapeutic use in vascular disorders, Drugs, 1993;45:259-94.

28. Porta R, Calvani AB, Pescador R, et al., Absorption through the peritoneum of the macromolecular profibrinolytic drug defibrotide in the rabbit, Semin Thromb Hemost, 1991;17:404-6.

29. Richardson PG, Elias AD, Krishnan A, et al., Treatment of severe veno-occlusive disease with defibrotide: compassionate use results in response without significant toxicity in a high-risk population, Blood, 1998;92:737-44.

30. Richardson PG, Murakami C, Jin Z, et al., Multi-institutional use of defibrotide in 88 patients after stem cell transplantation with severe veno-occlusive disease and multisystem organ failure: response without significant toxicity in a high-risk population and factors predictive of outcome, Blood, 2002; 100:4337-43.

31. Richardson PG, Smith AS, Kernan NA, et al., Results of the large prospective study on the use of defibrotide (OF) in the treatment of hepatic veno-occlusive disease (vod) in hematopoietic stem cell transplant (HSCT), early intervention improves outcome - updated results of a Treatment IND (T-IND) expanded access protocol, Blood, 2013:122:700.

32. Richardson PG, Soiffer RJ, Antin JH, et al., Defibrotide for the treatment of severe hepatic veno-occlusive disease and multiorgan failure after stem cell transplantation: a multicenter, randomized, dose-finding trial, Biol Blood Marrow Transplant, 2010;16:1005-17.

33. Richardson P, Tomblyn M, Kernan N, et al, Defibrotide (DF) in the treatment of severe hepatic veno-occlusive disease (VOD) with multi-organ failure (MOF) following stem celt (VOD) with multi-organ fallure (Mof) following stem cell transplantation (SCT): Results of a phase 3 study utilizing
historical control, Blood, 2009;114:Abstract 654.

34. Corbacioglu S, Cesaro S, Faraci M, et al., Defibrotide for prophylaxis of hepatic veno-occlusive disease in paediatric hremopoietic stem-cell transplantation: an open-label, phase 3, randomised controlled trial, Lancet, 2012:379:1301-9. 